\title{
Bemerkung zu dem Artikel des Hernn Comstock in Nr. 3821 der A. N.
}

Mir will scheinen, daß Herrn Comstocks interessante Notiz zu einer falschen Beurteilung meines Artikels in Nr. 3795 der A. N. Veranlassung geben könnte. Ich glaube doch, dab man aicht bestimmter die Zuverlässigkeit der gefundenen Zahlenwerte ablehnen und die Möglichkeit des Hervorgehens ganz andrer Werte aus einer größeren Zahl vod Beobachtungen zugeben kann, als ich dies mit den Worten (S. 40) getan habe:

- Im Übrigen bemerke ich nochmals ausdrücklich, daß die aogeführten Zahlen mehr des Beispiels wegen und nicht um sichere Resultate zu geben angeführt worden sind, denn zur Ableituog solcher müßten die Beobachtungen zahlreicher und über verschiedene Stundenwinkel verteilt sein.e
Ich mochte nir aun noch hinzuzufligen erlauben, daß mein Artikel aus Überlegungen hervorgegangen ist, die ich vor 11 Jahren angestellt habe. Darin mag eine Entschuldigung daftir liegen, da $\mathrm{B}$ ich die seitdein von andereo Astronomen uber denselben Gegeastand veroffentlichten Arbeiten übersehen habe. Ich hätte diese sonst selbstverstäadlich erwähnt.

Herr Comstock läßt übrigens bei seinen Bemerkungeo einen Umstand außer Acht, den ich besonders hervorgehoben habe, nämlich den, daß das Spektrum der Nova Persei sich sehr bald zu einem ganz eigenartigen entwickelte. Die von ihm angefübrten Zahlen dürfen deshalb nicht ohne weiteres bei den Beobachtungen der Nova zum Vergleiche herangezogen werden.

München, I 902 Okt. 23.

$$
\text { H. Seeliger. }
$$

\section{Photographische Aufnahmen des Planeten (420) Bertholda.}

Vor einigen Wochen fand ich zusaminen mit Herro Berberich den Planeten (420) Bertholda nachträglich alı zwei Platteo von Juni rgor, nämlich

$$
\begin{aligned}
& 1901 \text { Juni } 710^{\mathrm{h}} 53^{\mathrm{m}} \cdot 3 \text { M.Z. Kgst. } \alpha=17^{\mathrm{h}} 32^{\mathrm{m}} \cdot 3 \quad \delta=-21^{\circ} .0^{\circ} \text { Gr. I2.5 } \\
& 111121.0 \quad \circ \quad=1728.2 \quad=-205 \mathrm{r} \text {, }
\end{aligned}
$$

also ganz nahe arn Ephemeridenort. Der Planet ist mir damals entgangen, weil er zu nahe am Rande der Platte stand.

Astrophys. Observatorium Königstuhl, 1902 Sept. 22.

M. Wolf.

Beobachtungen des Planeten (19) Fortuna [1902 KE].

Auf der Sternwarte des Collegio Romano in Rom von Prof. E. Millosevich.

1902 olt. $30 \quad 10^{\mathrm{h}} 24^{\mathrm{m}} 3^{6^{\mathrm{s}}}$ t. m. Roma $\Delta \alpha=-3^{\mathrm{m}} 12^{5} .4^{8} \Delta d=-7^{\prime} 55^{\prime \prime} 7$ Cfr. 6.2 micr. filare ingr. 120 Gr. 10.0 $a$ app. $=3^{\mathrm{h}} 28^{\text {in }} 37^{\prime}: 31\left(9.450^{\mathrm{n}}\right) \delta$ app. $=+18^{\circ} 8^{\prime} 3^{\circ}: 3(0.595)$

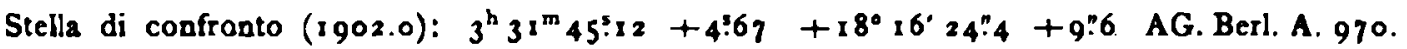

Auf der Sternwarte in Dusseldorf von Dr. W. Luther.

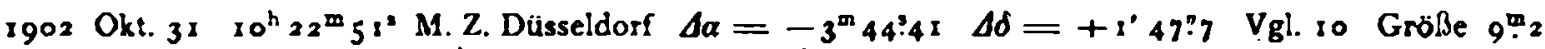

a app. $=3^{\mathrm{h}} 27^{\mathrm{m}} 4^{6: 72}\left(9.367_{\mathrm{n}}\right)$ o app. $=+18^{\circ} 4^{\circ} 22.9(0.709)$

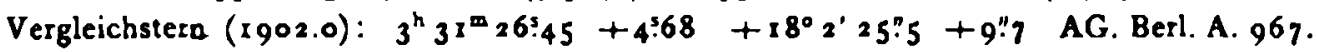

Literarischo Anzoige. F. Bidschof. Katalog von 2417 Sternen fur das mittlere Äquinoktium 1855.0 auf Grund der in den Bänden III, V, VIII und XI der Annalen der $k$. k. Universitätssternwarte zu Wien eathaltenen Meridiankreisbeobachtungen. Wien 1902. - Die in Rede stehenden Beobachtungen sind von 188 × bis 1889 angestellt und umfassen. im wesentlichen die Sterne der Santinischen Zonen vom Aquator bis - $10^{\circ}$ Dekl.

Planet (58) Concordia. Korr. der Ephemeride (Veröff. R. I. 18): Okt. $31+16^{3}+0: 9$. W. Luther.

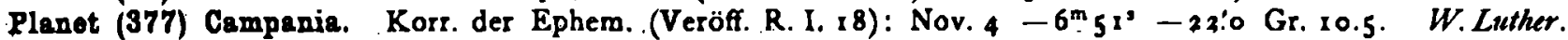

Pianota (416) Vaticana. Da una osservazione del Prof. Millosevich al Collegio Romano risulta: correzione alla. mia effemeride (A.N. 3816 ) pel 26 ottobre +0.12 +4.6; grand. 12.2. L'orbita dunque definitiva. G. Boctardi.

BD. +24:2733' and 27382. Prof. Hartwig macht mich darauf aufmerksam, da ${ }^{2}$ die große Eig. Bew. der beiden genannten Sterne (vgl. A. N. $3^{824}$ ) bereits von Herro F. A. Bellamy in M. N. Bd.60 p. 125 besprochen worden ist. Kr.

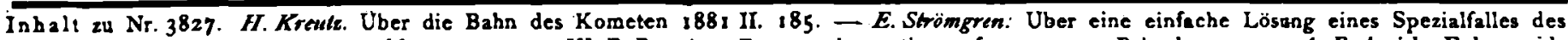
Dreikönerproblems. 191. - W. F. Denning. Recent observations of meteors at Bristol. 195. - A. Berberich. Ephemeride des Planeten (334) Chicago. 197. - Beobachtungen des Verandeilichen a Orionis (Ch. 2098). 197. - H. Seeliger. Bemerkung 2 dem Aitikel des Herm Comstock. in Nr. 3821 der A.N. ig9. - $M$. Wolf. Photographische Aufnahmen des Planelen (420) Bertholda. 199. - Beobachtungon des Planeten (19) Eortuna [1902 KE]. I99. - Literarische Anzeige. I99. Kleine Mitteilungen. 199. 